\title{
The Study of Factors Associated with Severity of In-Stent Restenosis in Patients Treated with PCI for Acute Coronary Syndromes
}

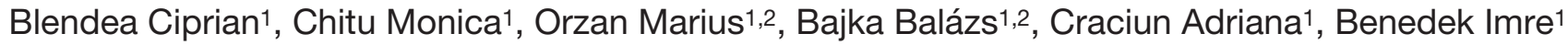 \\ 1 University of Medicine and Pharmacy of Tîrgu Mureș, Clinic of Cardiology, Tîrgu Mureș, Romania \\ 2 Cardio Med Medical Center, Department of Advanced Multimodal Imaging, Tîrgu Mureș, Romania
}

\begin{abstract}
Introduction: The management of in stent restenosis represents a topic of great actuality and interest, especially since the interventional treatment with stent implantation became largely accepted as the metod of choice in patients with acute coronary syndromes. Identification of certain risk factors that could predict the development of an in stent restenosis and its severity could be extremely useful for the clinical management of these patients. Methods: We retrospectively analyzed a total of 60 stent restenoses encountered in 57 patients admitted and treated in the Cardiology Clinic of Tirgu Mures. The interval of occurrence of restenosis ranged between 2 months and 37 months postintervention. We monitored the demographic characteristics (age, gender, colesterol, presence of renal insufficiency) and we realized a descriptive qualitative analysis of the angiographic procedural aspects. The in stent restenosis occurred most frequently on left anterior descending artery (63\%), followed by the circumflex artery $(22.15 \%)$ and right coronary artery (14.8\%), regardless of the degree of stenosis prior to revascularization. Results: Statistical analysis using Chi square test revealed no statistically significant differences in terms of the correlation between the incidence of restenosis and gender $(p=0.14)$, treatment with ACE inhibitors $(p=0.16)$, implanted stent diameter $(p=0.22)$ or the type of procedure (ram crossing over a secondary branch being considered as a procedure involved in the genesis of severe restenosis) ( $p=0.2)$. We used the t-student test for comparative analysis of the correlation between the continuous variables related to initial native lesion diameter and the degree of restenosis, without finding any a statistically significant correlation between them $(p=0.226)$. However, a statistically significant correlation was found between cholesterol levels and the degree of stenosis ( $p=0.039$ ). Descriptive analysis of restenosis lesions did not find any statistically significant correlation with the type or degree of stenosis in the native vessel, but showed statistically significant differences when evaluating the geometric assumption of restenosis by intraluminal diameter or intraluminal area ( $\mathrm{p}=0.0018)$, suggesting that assessment of the degree of restenosis should be performed only by planimetric area. Conclusions: We can conclude that in stent restenosis represents a plurifactorial phenomenon, that is not conditioned by the severity of the native lesion or by the administration of ACE inhibitors or Spironolactone, however it depends directly on the control of cholesterol values afther the coronary revascularization.
\end{abstract}

Keywords: in-stent restenosis, acute coronary syndromes, intraluminal diameter, intraluminal area, descriptive analysis

Received: 18 September 2015 / Accepted: 02 November 2015

\section{Introduction}

The number of percutaneous coronary angioplasty interventions associated with stenting has presented a significant increase and this technique tends to be the most used method of myocardial revascularisation [1].

Cardiac catheterization and selective coronary exploration started on another pathway since balloon angioplasty have been introduced by Andreas Gruntzing in 1977 $[2,3]$, leaving behind the area of the diagnosis procedures and advancing in the therapeutical one. The balloon angioplasty spred rapidly as a new therapy, however presenting the major limitation of the vascular recoil that could cause the abrupt closure of the vessel and restenosis. The pathophysiological mechanism of the restenosis could be explained by the negative vascular remodeling, the elastic recoil of the vessel and the thrombosis of the lesion, partially reduced by introduction of new antiplatelet therapy, leading to a restenosis rate below 30\% [4]. This fact led to the introduction of the bare metal stents, materials that

${ }^{*}$ Correspondence to: Ciprian Blendea

E-mail: morpheusadenom@yahoo.com could produce a radial force over the stented coronary segment, preventing the elastic recoil of the vessel. Altough the stents significantly reduced the restenosis rates, their use as a first choice therapy in coronary revascularization interventions led in short time to a new challenge - the issue of in stent restenosis. Altough the incidence of in-stent restenosis was smaller comparatively with the one noticed after the balloon angioplasty [5], it was still situated in the range between $15 \%$ and $30 \%$ [6].

The introduction of drug eluting stents (DES) resulted in a significant decrease in the number of in stent restenosis, however this phenomenon continues to persist even after the DES implantation, altough at a smaller rate comparatively with bare metal stents. Despite this fact, the great number of coronary revascularization interventions in parallel with the increased number of drug eluting stents implanted in the last years, underlines the global importance if the issue of in stent restenosis [7].

The aim of our study was to analyse the factors that could predict the development of an in stent restenosis and its severity, identification of these factors baing extremely useful for the clinical management of these patients. 


\section{Material and method}

We retrospectively analyzed a number of 60 in stent restenosis encountered in 57 patients who presented to the emergency room with an acute coronary syndrome. All patients presented with clinical, electrocardiographic and enzyme features of a STEMI acute myocardial infarction, and in all cases urgent coronary angiography was performed, which identified the site of the culprit lesion and was followed by stent implantation. All procedures was preceded by administration of optimal antiplatelet and anticoagulation therapy. The type of interventional treatment performed was selected in accordance to the angiographic status of the target lesion, for instance in case of coronary lesions with high calcium content, stenting was preceded by non-compliant coronary balloon expansion.

All patients benefited of optimal medical therapy from the moment of coronary revascularization for acute coronary syndrome, including aspirin $(75 \mathrm{mg})$, clopidogrel (600 mg loading dose followed by $150 \mathrm{mg} /$ day 7 days, then $75 \mathrm{mg} / \mathrm{day}$ ), angiotensin-converting enzyme inhibitors and statins $(80 \mathrm{mg}$ of atorvastatin in the first 30 days after the myocardial infarction, on the basis of local protocols).

In all patients we analysed demographic data, medical history and risk factors (age, gender, smoking status, diabetes, dyslipidemia, obesity, hypertension and biochemistry).

For the descriptive analysis of the initial target lesions of the native coronary vessels and for analysis of the in stent restenosis we used the soft Siemens QCA (quantitative coronary vessel analysis) (Fig.1).

Chi square test was used for the statiscal analysis of the demographic data, systemic factors related to the clinical status of the patient, the presence of comorbidities and the local factors related to the target lesion. We also analyzed the importance of the male sex in the occurence of in stent restenosis.

For the procedure of coronary revascularization we used bare metal stents implanted at maximal pressure. The coro-

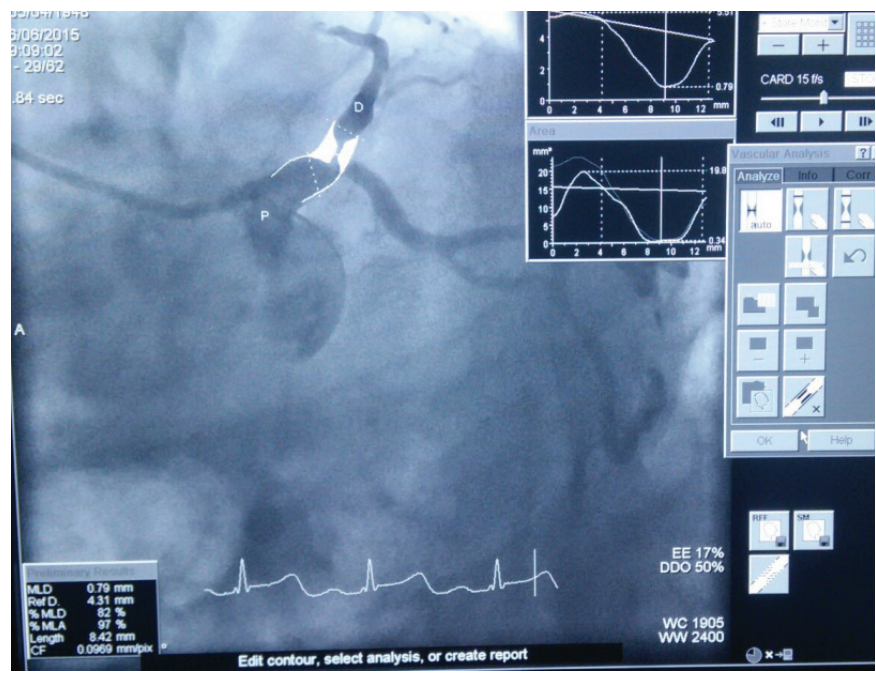

Fig. 1. The quantification of the degree of coronary stenosis based upon the QSA Siemens soft (with permision of the Clinic of Cardiology, Tîrgu Mureș, Romania) nary stents implanted had a diameter between $2.5 \mathrm{~mm}$ and $3.5 \mathrm{~mm}$. For 13 patients we used stens with diameter of $3.5 \mathrm{~mm}$, in 32 patients we used stents with the diameter of $3 \mathrm{~mm}$, in 6 patients we used stents with a diameter of $2.75 \mathrm{~mm}$ and in 4 patients we used stents with a diameter of $2.5 \mathrm{~mm}$. The lenght of the stent varied between 29 and $9 \mathrm{~mm}$. In 2 patients the implanted stents had a lenght of 9 $\mathrm{mm}$, in 5 patients $13 \mathrm{~mm}, 1$ patient received a stent of 14 $\mathrm{mm}$ length, 22 patients received a stent with a length of 15 $\mathrm{mm}$, in 14 patients we implanted a $18 \mathrm{~mm}$ length stent, in 5 patients a $23 \mathrm{~mm}$ stent, respectively longer stents (of 24 $\mathrm{mm}, 26 \mathrm{~mm}, 28 \mathrm{~mm}$ and $29 \mathrm{~mm}$ ) were implanted in one patient each.

The restenosis was classified in 4 types: type 1 represented by the focal restenosis, type 2 represented by the diffuse restenosis under $10 \mathrm{~mm}$; type III - diffuse restenosis which overcomes $10 \mathrm{~mm}$, with the eventual damage of the distal or proximal edges of the native vessel from the vicinity of the stent; respectively type IV represented by the total occlusion of the stent caused by the restenotic material (Fig. 2).

\section{Results}

From the 57 patients, 9 patients presented with unstable angina, 20 with non-STEMI acute myocardial infarction and 46 with ST-elevation acute myocardial infarction. The initial target lesion was identified at the level of all the coronary vessels, but the most frequent location was at the level of the Left Anterior Descending Artery (LAD). In $61.5 \%$ of cases the target lesion was located at the level of the left descending artery, in $21.15 \%$ at the level of the circumflex artery and in $17.3 \%$ of cases at the level of the right coronary artery. From this study population, 10 patients had a single coronary lesion, 36 patients presented significant lesions other than the coronary artery culprit lesion and 9 presented significant angiographic lesions on all the coronary branches, having a trivascular coronary artery disease. Fifteen patients presented complex lesions of

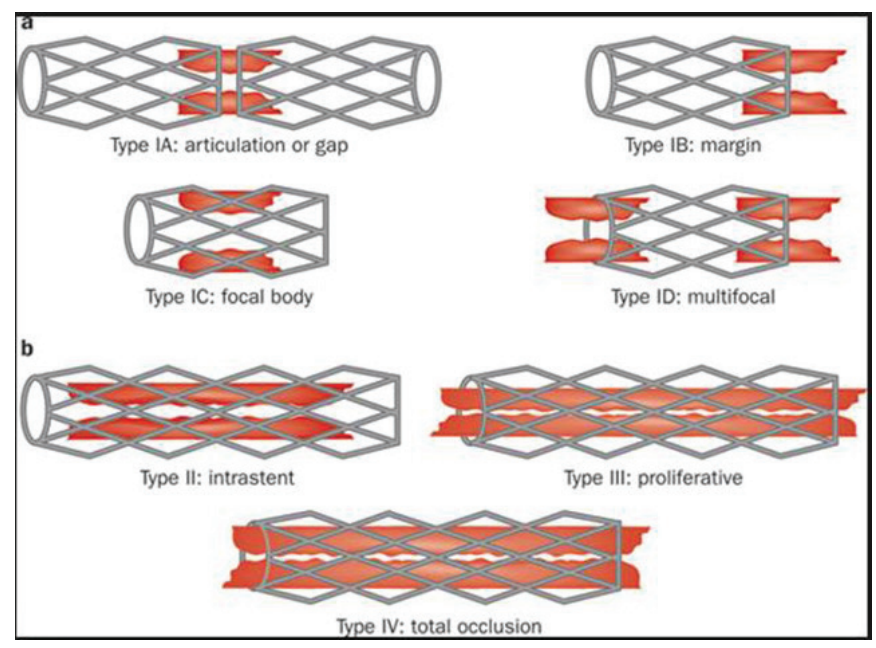

Fig. 2. Types of instent restenosis pattern 
bifurcation, with the necessity of implantation of the stent at the level of the main coronary branch over a secondary coronary branch, with a favorable angiographic aspect and maintaining the patency of the secondary branch at the end of the revascularization procedure.

In what concerns the time interval from the initial intervention until the occurence of the restenosis, the shortest time was of 2 months, and the longest one of 37 months.

The gender distribution was relatively homogeneous ( 24 women, respectively 31 men).

The great majority of patients presented in stent restenosis at the level of the LAD, followed by the CxA and RCA (Fig. 3).

Regarding the comorbidities, 5 patients presented atrial fibrilation, 6 patients uncontrolled drug hypertension, 17 patients diabetes (from whom 10 presented the uncontrolled type) and 38 patients presented dyslipidemia.

The administration of angiotensin-converting enzyme inhibitors, considered as a promoter of restenosis in clinical trials, it has not been proved in our study to have a role in the occurence of in stent restenosis in the analyzed patients $(\mathrm{p}=0.38)$.

The post revascularization ejection fraction $(\mathrm{EF})$ was analyzed, measured prior to hospital discharge, without finding any significant differences in this regard between the group with $\mathrm{EF}<50 \%$ and the group with $\mathrm{EF}>50 \%$ $(\mathrm{p}=0.59)$ (Fig. 4).

The presence of atrial fibrillation did not find any statistically significant correlation with the occurence of in stent restenosis $(\mathrm{p}=0.62)$.

Analysis of the pre-procedural TIMI flow did not show any difference between the group with TIMI flow 0 and the one with TIMI flow $>0(\mathrm{p}=0.38)$.

Regarding the systemic factors, we did not find any statistically significant assoiciation of in-stent restenosis incidence and the presence of diabetes or of high blood pressure values uncontrolled with drug treatment. On the contrary, cholesterol values of the revascularized patients immediately after the revascularization and at follow-up

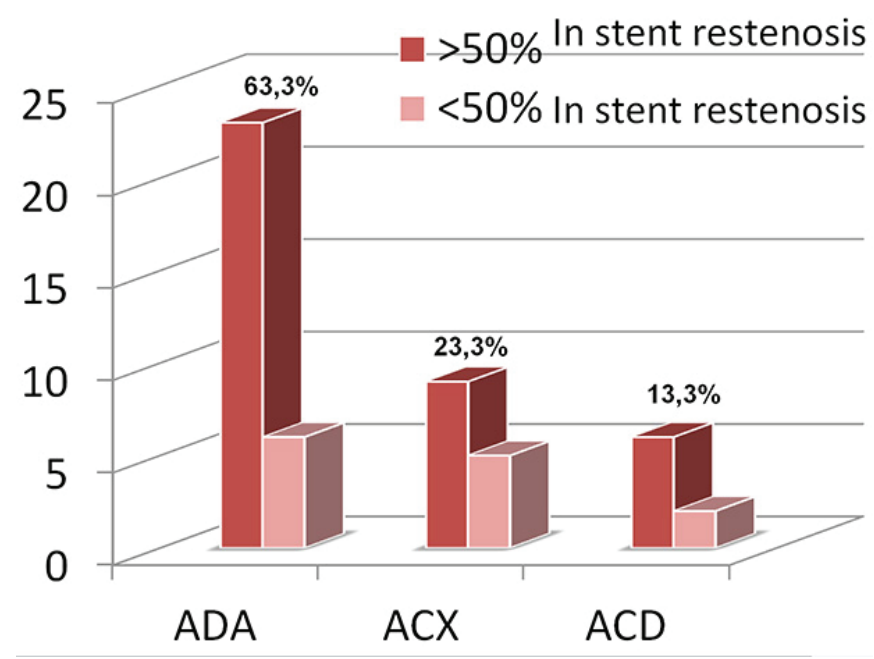

Fig. 3. The localisation of in stent restenosis showed a direct and statistically significant correlation with the degree of in stent restenosis demonstrated by imaging analysis ( $\mathrm{p}=0.039$ ) (Fig. 5).

At angiographic qualitative analysis, the initial periprocedural aspect did not show any significant difference between the groups regarding the frequency of restenosis and the diameter or length of the implanted stent, or the complexity of the procedures (in complex interventions requiring implantation of the coronary stent over a secondary branch, which is usually considered to be a procedure with a high risk for the occurence of a severe in stent restenosis) $(\mathrm{p}=0.23)$.

There were analyzed the diameter of the initial native lesion and the degree of in stent restenosis using the soft Siemens QCA for the comparative analysis and t Student test for the continuous variables, whitout succeeding to prove a significant correlation between these two.

\section{Discussions}

Despite the technological evolution, the technical improvement of the percutaneous coronary angioplasty and significant investments in the endovascular treatment field, the topic of in stent restenosis remains a great issue which rises great difficulties in the treatment of coronary ischemic diseases.

A large number of improvements of the endovascular prosthesis (either bare metal stents of first and 2nd generation, or the new drug eluting stents) have been encountered recently. At the same time, new technology have been designed for applying the farmacological substance on the metal matrix of the stent, with a large diversity of the active substance, while new schemes of drugs and significant improvement of the devices used in the endovascular treatment have significantly broadened the therapeutical horizont of the interventional cardiology.

Similar to other studies of in stent restenosis [8], despite the inclusion of all clinical, lesion related, and periprocedural factors known to influence the rate of restenosis, there there are still remaining some patient related aspects

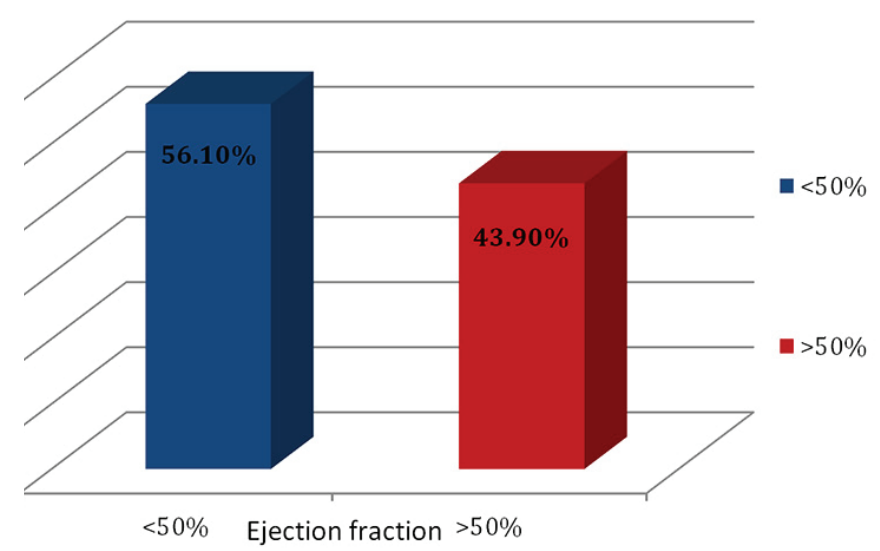

Fig. 4. The distribution of in stent restenosis based on the post revascularization ejection fraction 
witch contributes to this phenomenon. So, there are possible genetic variations in platelet function [9], angiotensinconverting enzyme activity related factors $[10,11]$, inflammatory response $[12,13]$, or alteration of one or more signal transduction pathways [14] that may be among these factors.

\section{Conclusions}

The phenomenon of in stent restenosis seems to be a plurifactorial one, apparently not influenced by the severity of the initial lesion or the features related to the procedure. The only parameter which had a statistically significance in our study is represented by the cholesterol value, which was significantly associated with the severity of restenosis.

\section{Acknowledgement}

This paper is supported by the Sectoral operational Programme Human Resources Development (SOP HRD), financed from the European Social Fund and by the Romanian Government under the contract number POSDRU/159/1.5/S/133377.

\section{References}

1. Mack MJ, Brown PP, Kugelmass AD, et al. Current statusand outcomes of coronary revascularization 1999 to 2002: 148,396 surgical and percutaneous procedures. Ann Thorac Surg. 2004;77:761-766.
2. Connors JJ 3rd, Wojak JC. Percutaneous transluminal angioplasty for intracranial atherosclerotic lesions: evolution of tehnique and short-term results. J Neurosurg 1999;91(3):415-23.

3. Gupta R, Schumacher HC, Mangla S, et al. Urgent endovascular revascularization for symptomatic intracranial atherosclerotic stenosis. Neurology 2003;61:1729-1735.

4. Moreno PR, Palacios IF, Leon MN, et al. Histopathologic comparison of human coronary in-stent and post-balloon angioplasty restenotic tissue. Am. J. Cardiol. 1999;84(4):462-6,A9

5. Serruys PW, Strauss BH, Beatt KJ, et al. Angiographic follow-up after placement of a self-expanding coronary-artery stent. N. Engl. J. Med. 1991;324(1):13-7

6. Serruys PW, Foley DP, Höfling B, et al.Carvedilol for prevention of restenosis after directional coronary atherectomy: Final results of the European carvedilol atherectomy restenosis (EUROCARE) trial. Circulation. 2000;101(13):1512-8.

7. Maisel WH. Unanswered questions - drug-eluting stents and the risk of late thrombosis. N. Engl. J. Med. 2007;356(10):981-4

8. Kastrati A, Mehilli J, Dirschinger J, et al. Restenosis after coronary placement of various stent types. Am J Cardiol 2001;87:34-9.

9. Chandrasekar B, Tanguay JF. Platelets and restenosis. J Am Coll Cardiol 2000;35:555-62.

10. Van Bockxmeer FM, Mamotte CD, Gibbons FA, et al. Angiotensinconverting enzyme and apolipoprotein E genotypes and restenosis after coronary angioplasty. Circulation 1995;92:2066-71.

11. Libby $P$, Tanaka $H$. The molecular bases of restenosis. Prog Cardiovasc Dis 1997;40:97-106.

12. Welt FG, Edelman ER, Simon DI, Rogers C. Neutrophil, not macrophage, infiltration precedes neointimal thickening in ballooninjured arteries. Arterioscl Thromb Vasc Biol 2000;20:2553-8.

13. Epstein SE, Speir E, Unger EF, et al. The basis of molecular strategies for treating coronary restenosis after angioplasty. J Am Coll Cardiol 1994;23:1278-88. 